\title{
Substrate screening effects on the quasiparticle band gap and defect charge transition levels in $\mathrm{MoS}_{2}$
}

\author{
Mit H. Naik and Manish Jain \\ Center for Condensed Matter Theory, Department of Physics, \\ Indian Institute of Science, Bangalore 560012, India
}

\begin{abstract}
Monolayer $\mathrm{MoS}_{2}$ has emerged as an interesting material for nanoelectronic and optoelectronic devices. The effect of substrate screening and defects on the electronic structure of $\mathrm{MoS}_{2}$ are important considerations in the design of such devices. We find a giant renormalization to the freestanding quasiparticle band gap in the presence of metallic substrates, in agreement with recent scanning tunneling spectroscopy and photoluminescence experiments. Our sulfur vacancy defect calculations using the DFT+GW formalism, reveal two CTLs in the pristine band gap of $\mathrm{MoS}_{2}$. The (0/-1) CTL is significantly renormalized with the choice of substrate, with respect to the pristine valence band maximum. The $(+1 / 0)$ level, on the other hand, is pinned $100 \mathrm{meV}$ above the pristine VBM for the different substrates. This opens up a pathway to effectively engineer defect charge transition levels in 2D materials through choice of substrate.
\end{abstract}




\section{INTRODUCTION}

$\mathrm{MoS}_{2}$, part of the family of the layered transition metal dichalcogenides (TMDC), has garnered great interest owing to its diverse applications in nanoelectronics and optoelectronics [1-3]. High current on-off ratios in field effect transistors as well as efficient valley and spin control with optical helicity have been achieved using $\mathrm{MoS}_{2}[4$, 5]. The direct band gap in monolayer $\mathrm{MoS}_{2}$ is exploited in building ultrasensitive phototransistors [6 8]. $\mathrm{MoS}_{2}$ is also considered a promising alternative to platinum as a catalyst in the hydrogen evolution reaction 9 [1].

Effect of the dielectric environment and defects on the electronic structure of $\mathrm{MoS}_{2}$ are the most important considerations in the design of devices using $\mathrm{MoS}_{2}$ [12 15]. Single layer $\mathrm{MoS}_{2}$, achieved through transfer post exfoliation or through direct epitaxial growth, [13, 16] is typically supported on a substrate. [16-20] Scanning tunneling spectroscopy (STS) measures the quasiparticle band gap of $\mathrm{MoS}_{2}$ on metallic substrates. [16, 21] The screening from the metal is consistently found to reduce the gap. [13, 16, 21,23] In the presence of graphene or graphite as substrate, a renormalization larger than $300 \mathrm{meV}$ is observed in the quasiparticle band gap of $\mathrm{MoS}_{2}$. [13, 16, 23, 27] Photoluminescence properties of $\mathrm{MoS}_{2}$ are also strongly influenced by the ambient dielectric environment. [16, 28] A red-shift in exciton peaks due to substrate screening effects is computed in Ref. [29].

The most abundant native defect found in monolayer $\mathrm{MoS}_{2}$ is the sulfur vacancy. [12] Sulfur vacancies induce states in the gap of pristine $\mathrm{MoS}_{2}$, thus affecting its electronic and optical properties. [9, 30, 31] Noise nanospectroscopy to probe the ionization dynamics of sulfur vacancy defects in $\mathrm{MoS}_{2}$ show 0 and -1 charge states of the defect to be stable. [32] Charged-impurity scattering from sulfur vacancies could thus be an important factor limiting the mobility of carriers in $\mathrm{MoS}_{2}$. [32] While effort is constantly made at attaining lower concentration of defects in $\mathrm{MoS}_{2}$, sulfur vacancies have found a favourable role in enhancing the rate of hydrogen evolution reaction. [9] Defect levels induced by sulfur vacancies in the band gap are responsible for the adsorption of hydrogen. [9, 33] The strength of hydrogen binding at the defect sites is determined by the difference in energy between the defect state and the Fermi level. [33] This binding is favourable for catalysis if the hydrogen is bound neither too strongly nor too weakly. 34] Pathways to engineer the position of the defect level in the gap are thus vital to enhance the hydrogen evolution reaction. [9, 35] 
A number of theoretical calculations, based on first principles density functional theory (DFT), [36, 37] have been carried out to study sulfur vacancies in monolayer $\mathrm{MoS}_{2}$. [30, 3842. Calculation of charged defects and in turn the charge transition levels (CTLs) within DFT has pitfalls owing to the underestimation of the band gap in Kohn-Sham DFT. [43] Some simulations use hybrid functionals, as proposed by Heyd, Scusseria and Erzernhof (HSE), [44] as an attempt to overcome the band gap problem [30, 38]. However, the band gap of monolayer $\mathrm{MoS}_{2}$ computed using HSE is about $2.2 \mathrm{eV}$, [30, 38, 45, 46] which is $0.5 \mathrm{eV}$ smaller than the experimentally measured quasiparticle band gap of free-standing $\mathrm{MoS}_{2}$. [4] This could affect the results on the defect CTLs in $\mathrm{MoS}_{2}$. Furthermore, substrate screening effects can not be effectively studied using DFT or hybrid functionals. [48]

Many body perturbation theory in the GW approximation has been combined with DFT in the well known DFT+GW formalism [49, 50] and has been used to predict accurate defect formation energies and CTLs. [51 53] In the DFT+GW formalism, the energy associated with atomic relaxations on adding an electron is taken into account at the DFT level, and the quasiparticle excitation energy at the GW level. Performing GW calculations on transition metal dichalcogenides (TMDCs) in particular are computationally challenging owing to the stringent convergence parameters. [54 56] A DFT+GW study on defects in TMDCs, which entail super cell calculations, require massive computation. Additionally, the effect of substrate screening can be taken into account accurately within the GW approximation. [48, 55, 57, 58] While it is known that metallic substrates renormalize the pristine quasiparticle band gap significantly, it isn't apparent if the defect levels will continue to prevail in the pristine gap or be pushed above or below the pristine CBM or VBM, respectively.

In this work, we study the effect of substrate screening on the quasiparticle band gap and defect charge transition levels in monolayer $\mathrm{MoS}_{2}$. We have considered graphene, hexagonal $\mathrm{BN}$, graphite and $\mathrm{SiO}_{2}$ as substrates. At the DFT level, we find that these substrates do not influence the electronic structure of $\mathrm{MoS}_{2}$. This is due to the absence of long range correlation effects in DFT. At the GW level, however, we find a significant renormalization in the quasiparticle band gap in the presence of these substrates. The quasiparticle gap is renormalized from its free-standing value of $2.7 \mathrm{eV}$ to $2.4 \mathrm{eV}$ in the presence of graphene and to $2.2 \mathrm{eV}$ in the presence of graphite as substrate. In the presence of $\mathrm{BN}$ or $\mathrm{SiO}_{2}$, the gap is close to that of free-standing $\mathrm{MoS}_{2}$. These results are in good agreement with recent experimental measurements. [13, 16, 21, 47, 59 62] We also study the electronic structure 
of $\mathrm{MoS}_{2}$ in the presence of sulphur vacancy defects. The sulfur vacancy induces states in the pristine band gap of $\mathrm{MoS}_{2}$. We compute the CTLs of the sulfur vacancy using the DFT+GW formalism. Two CTLs appear in the quasiparticle gap: the $(+1 / 0)$ and $(0 /-1)$ levels are $0.1 \mathrm{eV}$ and $2.2 \mathrm{eV}$ above the pristine valence band maximum (VBM), respectively. We further study the effect of substrate screening on the CTLs. The $(+1 / 0)$ level lies within $100 \mathrm{meV}$ of the VBM in the presence of substrates as well. The (0/-1) level, on the other hand, is significantly renormalized and can be tuned with the choice of substrate.

\section{COMPUTATION DETAILS}

The density functional theory (DFT) calculations are performed using the plane-wave pseudopotential package Quantum Espresso. [63] We use the local density approximation for the exchange correlation functional and norm-conserving pseudopotentials. The wavefunctions are expanded in plane waves upto an energy cut off of $250 \mathrm{Ry}$. For the unit cell $\mathrm{MoS}_{2}$ calculations, the cell dimension in the out-of-plane direction is fixed at $35 \AA$ and the Brillouin zone sampled with a $12 \times 12 \times 1 \mathrm{k}$-point grid. The relaxed in-plane lattice parameter of $\mathrm{MoS}_{2}$ is $3.15 \AA$. We simulate a sulfur vacancy in $\mathrm{MoS}_{2}$ by constructing a $5 \times 5$ in-plane super cell and removing a sulfur atom. The cell dimension in the out-of-plane direction here is fixed at $18 \AA$. K-point sampling of $2 \times 2 \times 1$ is used in the super cell calculations. The formation energy of charged sulfur vacancies computed at the DFT level need to be corrected for the spurious electrostatic interaction between the charge and its periodic images. The electrostatic corrections are computed using the CoFFEE code. [64]

The quasiparticle excitation energies are computed using the BerkeleyGW code. 65 67] For the unit cell $\mathrm{MoS}_{2}$ calculation, we use a k-point sampling of $24 \times 24 \times 1$ and 8,400 valence and conduction states. We find the quasiparticle band gap of monolayer $\mathrm{MoS}_{2}$ to be 2.7 $\mathrm{eV}$, which is in good agreement with previous calculations and experimental measurements. [24, 47, 54, 68] For the super cell calculations, we use a k-point sampling of $2 \times 2 \times 1$ and 19,000 valence and conduction states. We find that these parameters are sufficient to converge the gap at the $K$ point in the Brillouin zone to within $0.2 \mathrm{eV}$. The first 9,000 lowest energy wavefunctions are treated using a plane-wave energy cut off of $150 \mathrm{Ry}$, and the rest 10,000 wavefunctions are treated using a smaller cut off of 50 Ry. We find that this method produces accurate results at significantly reduced computation time. The states with low 
energy, below the Fermi level, are spatially localised. These gradually become more planewave like at higher energies. The higher energy wavefunctions can thus be described with a fewer number of plane-waves. We have tested this method using the unit cell $\mathrm{MoS}_{2}$. We find that the GW band gap is unchanged if we use the first 60 wavefunctions at 150 Ry cut off and the rest at 50 Ry cut off. It is worthwhile to note that the wavefunctions generated with the 50 Ry cut off need to be orthonormalized to the 150 Ry cut off wavefunctions. This is done in the following manner for the defect supercell calculations. We first generate 9,000 lowest energy wavefunctions at an energy cut off of 150 Ry. We then generate 19,000 lowest energy wavefunctions with an energy cut off of $50 \mathrm{Ry}$. We compute the overlap of these 19,000 with the original 9,000 bands. The bands with a large overlap with the original wavefunctions are left out, and the remaining are orthonormalized with the original. [69] We use PRIMME [70, 71] to generate the 9,000 bands at 150 Ry cut off and ScaLAPACK [72] exact diagonalization routines to generate the 19,000, 50 Ry cut off wavefunctions. Further, the static remainder technique [73] is used to accelerate convergence of the calculation with the number of empty states. A dielectric cut off of 35 Ry is used. The Coulomb interaction along the out-of-plane direction is truncated for the computation of dielectric matrix and self energy. [74] The dielectric function is extended to finite frequencies using the HybertsenLouie generalized plasmon pole (GPP) model. [67]

The substrates included in our calculations are $\mathrm{BN}, \mathrm{SiO}_{2}$, graphene, bilayer graphene (BLG), trilayer graphene (TLG) and graphite. The wavefunction cut-off used is 70 Ry for BN and $\mathrm{SiO}_{2}$; and 60 Ry for graphene, BLG, TLG and graphite calculations. K-point sampling used for graphene, BLG and TLG is $21 \times 21 \times 1$. K-point sampling used for graphite is $21 \times 21 \times 10$. K-point sampling used for $\mathrm{BN}$ and $\mathrm{SiO}_{2}$ is $15 \times 15 \times 1$ and $14 \times 14 \times 14$, respectively. For the 2D substrates, the cell dimension in the out-of-plane direction is chosen to match that of $\mathrm{MoS}_{2}$. We use the semiempirical Grimme [75] scheme to account for the van der Waals interactions between the layers in heterostructures constructed at the DFT level to obtain the interlayer spacings. We perform calculations on the unit cells of substrates to obtain their irreducible polarizabilities. The k-point sampling in the polarizability calculations is the same as those for the DFT calculations. The dielectric cut off used for graphene, BLG, TLG and graphite is $10 \mathrm{Ry}$. For BN and $\mathrm{SiO}_{2}$, the dielectric cut off used is $12 \mathrm{Ry}$ and 10 Ry, respectively. The number of unoccupied states for graphene, BLG, TLG and graphite is 250. For $\mathrm{BN}$ and $\mathrm{SiO}_{2}$, the number of states is 600 and 300, respectively. For metallic 
(a)

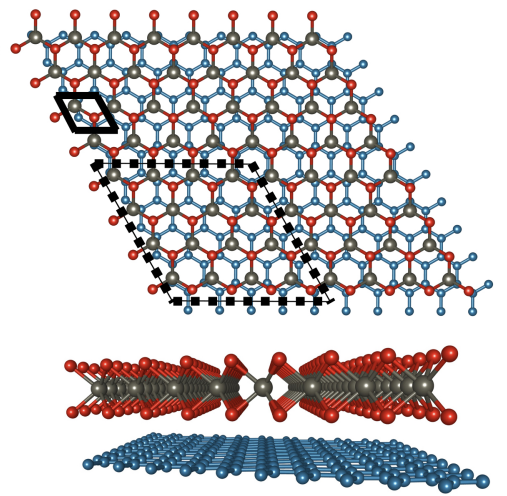

(e)

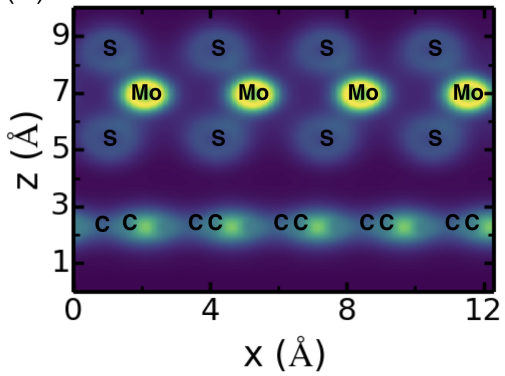

(b)

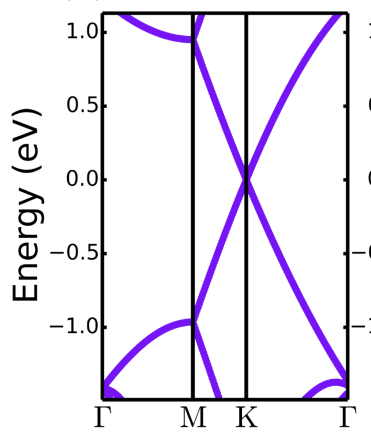

(c)

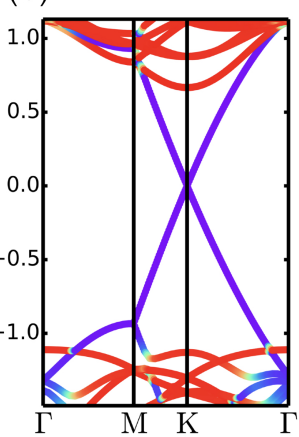

(d)

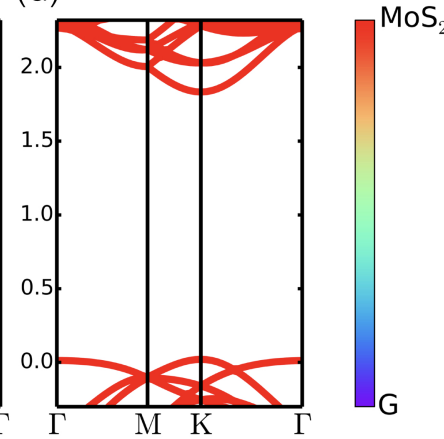

(g) (f)

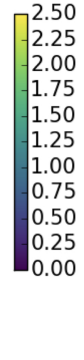

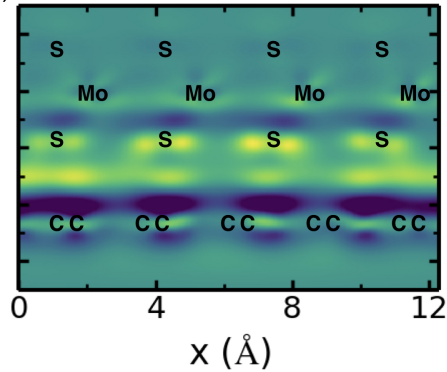

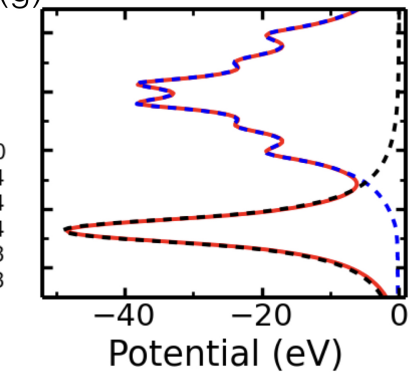

FIG. 1. (a) Single layer $\mathrm{MoS}_{2}$ on a graphene substrate, top-view and side-view. The black solid line marks the unit cell of $\mathrm{MoS}_{2}$. The dotted line marks the lattice-matched super cell used to perform the DFT calculations. (b), (c) and (d) DFT band structures of free-standing $5 \times 5$ super cell of graphene, lattice matched graphene- $\mathrm{MoS}_{2}$ heterostructure and free-standing $4 \times 4$ super cell of $\mathrm{MoS}_{2}$, respectively. The colors indicate the projected weights of the heterostructure wavefunctions onto the free-standing layers. (e) The charge density of the $\mathrm{MoS}_{2}$-graphene heterostructure, in e/ $\AA^{3}$, averaged along one of the in-plane lattice directions. (f) The charge density difference, between the $\mathrm{MoS}_{2}$-graphene heterostructure and the corresponding free-standing layers. (g) The potential of the $\mathrm{MoS}_{2}$-graphene heterostructure (red line), the free-standing graphene and the free-standing $\mathrm{MoS}_{2}$ layer (blue line).

substrates, the polarizability at the q-point close to the $\Gamma$ point is computed with a finer k-point sampling of $80 \times 80 \times 1$. 

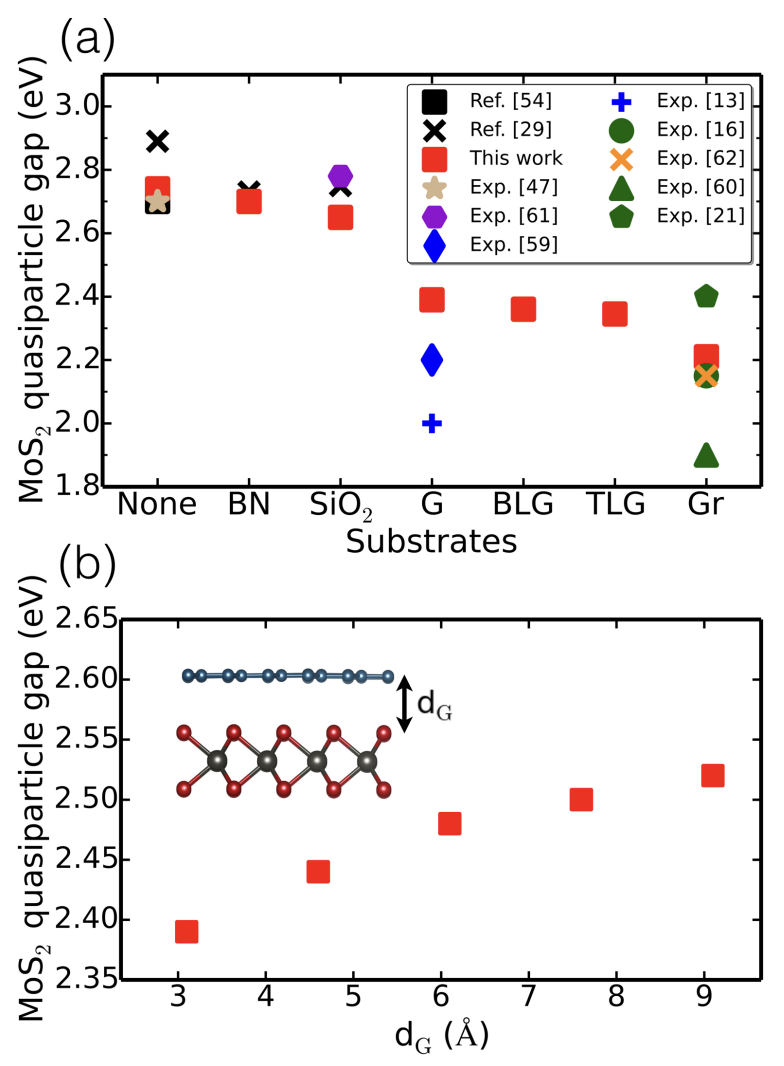

FIG. 2. (a) Quasiparticle band gap of $\mathrm{MoS}_{2}$, free-standing, and in the presence of monolayer $\mathrm{BN}$, bulk $\mathrm{SiO}_{2}$, graphene $(\mathrm{G})$, bilayer graphene (BLG), trilayer graphene (TLG) and graphite substrates. Experimental measurements of the quasiparticle gap in these systems are also shown in the plot. (b) Quasiparticle band gap of $\mathrm{MoS}_{2}$ in the presence of graphene as a function of increasing inter-layer spacing, $\mathrm{d}_{\mathrm{G}}$.

\section{EFFECT OF SUBSTRATE SCREENING}

We study the interaction between $\mathrm{MoS}_{2}$ and a substrate at the DFT level by constructing commensurate super cells that accommodate the two materials with a strain of less than $2 \%$. We use a $4 \times 4$ super cell of $\mathrm{MoS}_{2}$ and a $5 \times 5$ super cell of graphene. Fig. 1 (a) shows $\mathrm{MoS}_{2}$ on a graphene substrate. A similar geometry is used for the case of $\mathrm{MoS}_{2}$ on $\mathrm{BN}$ since the lattice parameter of $\mathrm{BN}$ is close to that of graphene. The $\mathrm{BN}$ or graphene layers are strained to attain a commensurate super cell. The relaxed interlayer spacing for the $\mathrm{MoS}_{2}$-graphene heterostructure is 3.1 A. Fig. 1 (b) and Fig. 1 (d) show the DFT band structure of the $5 \times 5$ super cell of graphene and the $4 \times 4$ super cell of $\mathrm{MoS}_{2}$, respectively. Fig. 1 (c) shows the band structure of the $\mathrm{MoS}_{2}$-graphene heterostructure. The DFT 
wavefunction of the heterostructure, for a given band and k-point, has been projected onto the wavefunctions of free-standing graphene and free-standing $\mathrm{MoS}_{2}$. The projected weights are then portrayed using a color map. Note that the energy of the VBM has been set to zero in these plots. It can be seen that interlayer coupling or hybridization is absent at the VBM and CBM of $\mathrm{MoS}_{2}$ in the heterostructure. At the DFT level, the band gap of $\mathrm{MoS}_{2}$ in the presence of graphene is unchanged. This is different from bilayer $\mathrm{MoS}_{2}$ where the overlap of wavefunctions of similar energies leads to strong hybridization and a transition of the gap from direct to indirect [76]. Slight hybridization is, however, seen far from the Fermi level, leading to the creation of small gaps in graphene of about $70 \mathrm{meV}$. These minigaps have been recently observed in $\mathrm{MoS}_{2}$-graphene heterostructures using angleresolved photoemission spectroscopy (ARPES). [77, 78] Fig. 11 (e) plots the charge density of the $\mathrm{MoS}_{2}$-graphene heterostructure, $\rho^{\mathrm{MG}}(\mathbf{r})$, averaged along one of the in-plane lattice vectors. Fig. 1 (f) plots the charge density difference, $\rho^{\mathrm{MG}}(\mathbf{r})-\rho^{\mathrm{M}}(\mathbf{r})-\rho^{\mathrm{G}}(\mathbf{r})$, in the same manner. In the heterostructure, the electronic charge density within each layer is slightly rearranged, but there is no possibility of charge transfer from one layer to the other due to the sizeable energy difference between the graphene Fermi level and the $\mathrm{MoS}_{2} \mathrm{CBM}$. Our Bader charge analysis further supports the absence of charge transfer. The directionality of the rearrangement of charges, leading to the formation of out-of-plane dipole moments, is explained by the non-uniform potential gradient induced in one layer due to the other (Fig. $1(\mathrm{~g}))$. At the equilibrium spacing, the potential from one layer is finite and decreasing in the vicinity of the other layer. This gradient acts like an effective electric field for the other layer leading to the rearrangement of electrons.

Performing GW calculations on the various super cell geometries is computationally demanding. We instead perform separate unit cell calculations on $\mathrm{MoS}_{2}$ and the substrates. To take into account the effect of a substrate on $\mathrm{MoS}_{2}$ we map the in-plane $\vec{q}+\vec{G}$ vectors of the $\mathrm{MoS}_{2}$ irreducible polarizability, $\chi_{\vec{q}}^{\vec{G} \vec{G}^{\prime}}$, to $\vec{q}+\vec{G}$ vectors of the substrate irreducible polarizability. The substrate polarizability element corresponding to the mapped $\vec{q}+\vec{G}$ vector is then added to the polarizability element of $\mathrm{MoS}_{2}$. [55, 57, 58] Using this method, the band gap reduction is slightly overestimated for bulk substrates. Fig. 2 (a) shows the quasiparticle band gap of $\mathrm{MoS}_{2}$ in the free-standing case and in the presence of $\mathrm{BN}, \mathrm{SiO}_{2}$, graphene $(\mathrm{G})$, bilayer graphene (BLG), trilayer graphene (TLG) and graphite (Gr) substrates. Also marked in the figure are the experimentally measured quasiparticle band gaps of $\mathrm{MoS}_{2}$ on 
these substrates. A significant renormalization to the band gap of $\mathrm{MoS}_{2}$ is captured at the GW level, while the gap remains unchanged at the DFT level. This is due to the inclusion of image charge effects at the GW level. The more metallic nature of the substrate, larger the band gap renormalization. A similar trend is observed for molecules on a metal substrate, where DFT fails to predict any renormalization to the molecular levels. While GW effectively captures the non-local screening due to image charge effects and shows a renormalization, in agreement with experimental findings. [79 81] The renormalization of the $\mathrm{MoS}_{2}$ quasiparticle band gap in the presence of $\mathrm{BN}$ and $\mathrm{SiO}_{2}$ is $40 \mathrm{meV}$ and $90 \mathrm{meV}$ respectively. In the presence of graphene, BLG, TLG and graphite; the renormalization is 350 $\mathrm{meV}, 380 \mathrm{meV}, 400 \mathrm{meV}$ and $530 \mathrm{meV}$ respectively. Our result for the renormalization in the presence of graphene is in good agreement with a recent GW calculation on the explicit $\mathrm{MoS}_{2}$-graphene heterostructure. [24] The value of the quasiparticle band gap measured experimentally is in excellent agreement for the free-standing case and in the presence of $\mathrm{SiO}_{2}$ substrate (Fig. 2 (a)). The experimental quasiparticle band gap of $\mathrm{MoS}_{2}$ measured in the presence of graphene and graphite substrate, on the other hand, is varied and falls in the range of 1.9 to $2.4 \mathrm{eV}$ (Fig. 2 (a)). In Fig. 2 (a), all the experimental values reported are measured using scanning tunneling spectroscopy except the one on $\mathrm{SiO}_{2}$ substrate, which uses photoluminescence excitation spectroscopy. We additionally study the effect of $\mathrm{MoS}_{2^{-}}$ graphene interlayer spacing on the quasiparticle band gap of $\mathrm{MoS}_{2}$. We find that the gap is sensitive to the spacing and can be tuned from $2.4 \mathrm{eV}$ to $2.5 \mathrm{eV}$ (Fig. 2 (b)). We estimate the error in our calculation of the quasiparticle band gap is $100 \mathrm{meV}$ in the case the 2D substrates and $150 \mathrm{meV}$ in the case of bulk substrates. There exist other factors in the experiment that could lead to further renormalization of the band gap in $\mathrm{MoS}_{2}$. These include the effect of carrier-induced plasmons in the system, which have recently been shown to close the gap by upto $150 \mathrm{meV}$. [61, 82] Additional screening from the metallic tip of the scanning tunneling microscope could also further renormalize the band gap. 83]

\section{SULFUR VACANCY DEFECT}

Fig. 3 (a) shows the DFT band structure of $5 \times 5$ super cell of $\mathrm{MoS}_{2}$ with a sulfur vacancy defect. Three defect states are induced in the gap on introducing the vacancy: one filled (indicated by green) bonding state and two degenerate unfilled (indicated by blue) anti- 

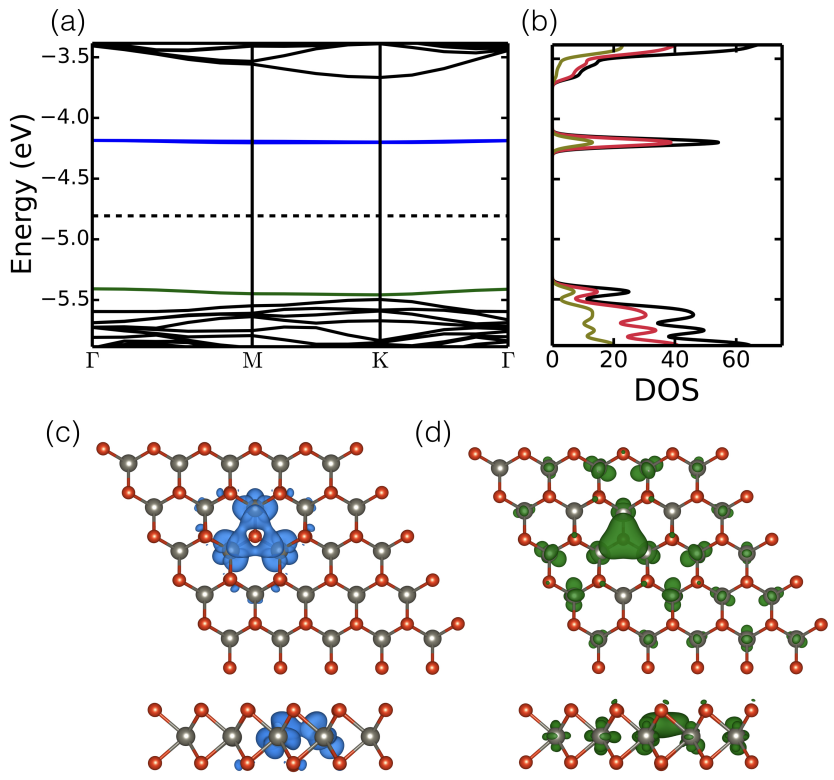

FIG. 3. (a) DFT computed band structure of a sulfur vacancy defect in a $5 \times 5$ super cell of $\mathrm{MoS}_{2}$. Three defect states are induced in the gap. The filled defect level is indicated in green, the unfilled levels are doubly degenerate and indicated in blue. The black dashed line marks the Fermi level. (b) Partial density of states (DOS) of the $5 \times 5$ super cell of $\mathrm{MoS}_{2}$ with a sulfur vacancy. The red line shows the Mo- $d$ contribution and the green line shows the S- $p$ contribution to the total density of states (black). (c) and (d) Isosurface of the defect levels induced in the gap of $\mathrm{MoS}_{2}$. The wavefunction plotted in blue is the corresponding unfilled defect level in the band structure and the one plotted in green is the filled defect level. The top view as well as the side view are shown.

bonding states. The charge density associated with these defect states is shown in Fig. 3 (c) and $(\mathrm{d})$. The empty states are localised over a smaller region in the material as compared to the filled state. These defect states are dominantly of the Mo-d character (Fig. 3 (b)). We compare the VBM and CBM of the pristine $\mathrm{MoS}_{2}$ system, and the defect levels with respect to the vacuum level as computed within DFT and GW. Fig. 4(a) and (b) shows a schematic of this comparison. We find that the DFT calculated CBM and the GW calculated CBM differ by about $0.1 \mathrm{eV}$, while the respective VBMs are different by $1 \mathrm{eV}$. Interestingly, the empty defect levels are found to line up. The filled defect level on the other hand remains shallow and close to the VBM. It has been shown that the CTLs of bulk systems line up between DFT and GW with respect to the average electrostatic potential in the system. 


\begin{tabular}{crrrr}
\hline \hline BZ sampling & $24 \times 24 \times 1$ & $12 \times 12 \times 1$ & $12 \times 12 \times 1$ & $10 \times 10 \times 1$ \\
$\mathrm{~N}_{b}$ & 6000 & 6000 & 750 & 750 \\
\hline Gap at $\mathrm{K}(\mathrm{eV})$ & 2.74 & 2.79 & 2.78 & 2.85 \\
Gap at $\Gamma(\mathrm{eV})$ & 4.02 & 4.06 & 4.08 & 4.13 \\
\hline \hline
\end{tabular}

TABLE I. Convergence of the band gap as a function of Brillouin zone sampling and number of bands.

(a)

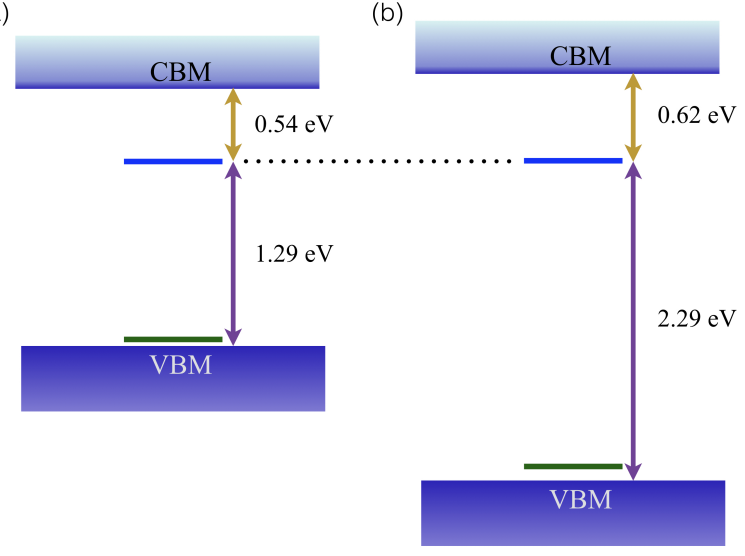

FIG. 4. (a) Schematic of the DFT prisitine VBM, pristine CBM and the defect levels, plotted with respect to the vacuum level. (b) Schematic of the GW pristine VBM, pristine CBM and the defect levels, plotted with respect to the vacuum level. The dotted line is a guide to the eye, showing that the unfilled defect levels line up between DFT and GW levels of theory.

[53] Here, we find that the defect levels line up with respect to the vacuum level, while the CTLs do not (Fig. 4).

The formation energy of a sulfur vacancy in charge state $q$ is given by,

$$
\begin{array}{ll}
\mathrm{E}_{q}^{f}\left[\overrightarrow{\mathrm{R}}_{q}\right]\left(E_{F}\right)=\left\{\mathrm{E}_{q}^{\mathrm{tot}}\left[\overrightarrow{\mathrm{R}}_{q}\right]+\mathrm{E}_{q}^{\text {corr }}\right\}-\mathrm{E}_{\text {pristine }} & \\
& +q\left\{\epsilon_{\mathrm{vbm}}^{\text {pristine }}+E_{F}-\Delta V_{0 / p}\right\}-\mu_{S}
\end{array}
$$

where $\mathrm{E}_{q}^{\mathrm{tot}}\left[\overrightarrow{\mathrm{R}}_{q}\right]$ refers to the total energy of the $5 \times 5$ super cell of $\mathrm{MoS}_{2}$ containing the defect in charge state $q . \overrightarrow{\mathrm{R}}_{q}$ refers to the relaxed atom positions in the super cell of the defect system in charge state $\mathrm{q} . \mathrm{E}_{q}^{\mathrm{corr}}$ is the electrostatic correction term to account for the spurious interaction of the charged defect with its periodic images. This term is zero for the case of the neutral defect. $\mathrm{E}_{\text {pristine }}$ is the total energy of a pristine super cell of $\mathrm{MoS}_{2}$ 

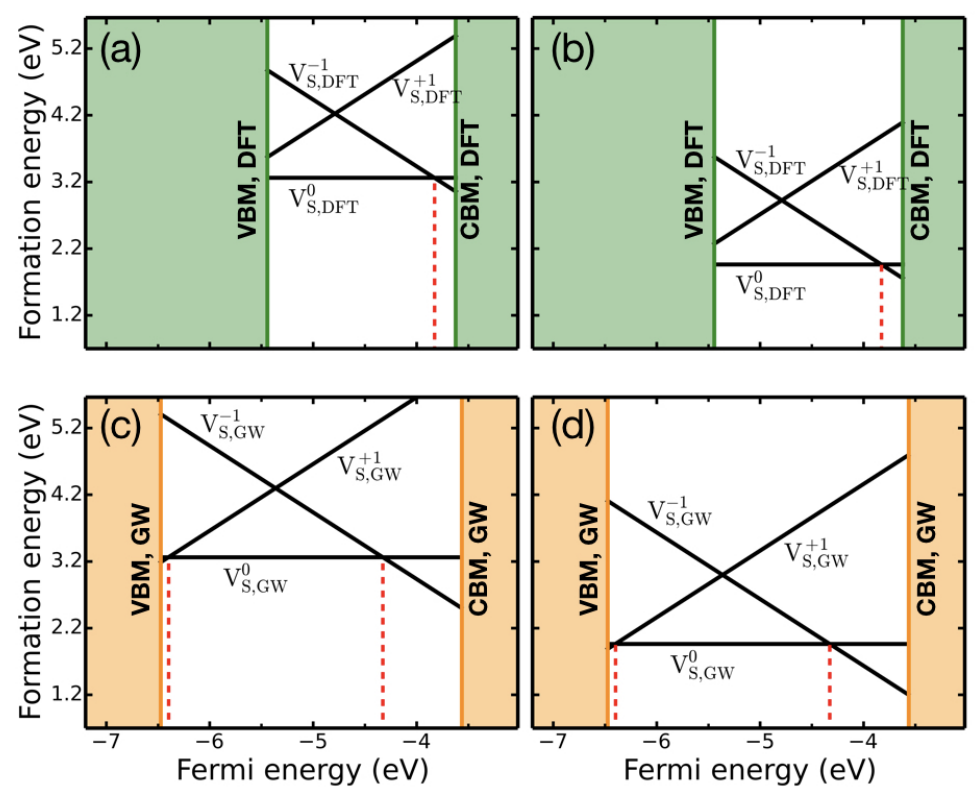

FIG. 5. Formation energy of the sulfur vacancy in different charge states as a function of the Fermi level. The Fermi level is taken to scan the energy range between the pristine VBM and CBM. (a) and (b) Computed at the DFT level, for sulphur rich and sulphur poor conditions respectively. (c) and (d) Computed using the DFT+GW formalism, for sulphur rich and sulphur poor conditions respectively. The charge transition levels that appear in the gap are marked with red dashed lines.

of the same size. The formation energy is a function of the Fermi level with respect to the VBM of the pristine system, $\epsilon_{\mathrm{vbm}}^{\mathrm{pristine}}+E_{F} . \Delta V_{0 / p}$ is the potential alignment term found by comparing the elecrostatic potential of the neutral defect cell and pristine cell, far from the defect. $\mu_{S}$ is the chemical potential of the sulfur atom removed from the pristine system to form the vacancy defect. This reference can be chosen to simulate sulfur-rich or sulfur-poor ambient conditions. For the sulfur-rich conditions, the chemical potential is chosen from the cyclo- $\mathrm{S}_{8}$ allotrope of sulfur. For sulfur-poor conditions, the chemical potential is chosen 1.3 $\mathrm{eV}$ below, the potential at which $\mathrm{MoS}_{2}$ is reduced to body-centered cubic (BCC) Mo metal [84, 85]. Fig. 5 (a) and (b) plot the DFT computed formation energy of the sulfur vacancy in $0,-1$ and +1 charge states as a function of the Fermi level. The Fermi level scans the pristine $\mathrm{MoS}_{2}$ gap. The formation energy here is determined following Eqn. 1 using the DFT computed total energy differences. The electrostatic correction term is determined to be $0.1 q^{2} \mathrm{eV}$, where $q$ is the charge state of the vacancy. Charge transition level, the Fermi level at which the formation energy of one charge state of the defect is equal to that of 
another is given by:

$$
\varepsilon^{\mathrm{q} / \mathrm{q}-1}=\mathrm{E}_{\mathrm{q}-1}^{f}\left[\overrightarrow{\mathrm{R}}_{\mathrm{q}-1}\right]\left(E_{\mathrm{F}}=0\right)-\mathrm{E}_{\mathrm{q}}^{f}\left[\overrightarrow{\mathrm{R}}_{\mathrm{q}}\right]\left(E_{\mathrm{F}}=0\right)
$$

The only charge transition level stable in the gap at the DFT level is $\varepsilon^{0 /-1}=1.6 \mathrm{eV}$ from the VBM. This is in good agreement with previous calculations [30, 38].

Within the DFT+GW formalism, the expression for the charge transition level can be rewritten into two parts. One that involves adding an electron to the system, and the other that takes into account the lattice relaxation effects due to to the added electron [52]. The former is evaluated as a quasiparticle excitation at the GW level and the latter is evaluated at the DFT level.

$$
\begin{aligned}
\varepsilon^{\mathrm{q} / \mathrm{q}-1} & =\left(\mathrm{E}_{\mathrm{q}-1}^{f}\left[\overrightarrow{\mathrm{R}}_{\mathrm{q}-1}\right]-\mathrm{E}_{\mathrm{q}-1}^{f}\left[\overrightarrow{\mathrm{R}}_{\mathrm{q}}\right]\right)+\left(\mathrm{E}_{\mathrm{q}-1}^{f}\left[\overrightarrow{\mathrm{R}}_{\mathrm{q}}\right]-\mathrm{E}_{\mathrm{q}}^{f}\left[\overrightarrow{\mathrm{R}}_{\mathrm{q}}\right]\right) \\
& =\mathrm{E}_{\text {relax }}+\mathrm{E}_{\mathrm{QP}}
\end{aligned}
$$

For the $\varepsilon^{0 /-1}$ evaluated using the DFT+GW formalism, we find $\mathrm{E}_{\mathrm{QP}}=2.3 \mathrm{eV}$ and $\mathrm{E}_{\mathrm{relax}}=$ $-0.1 \mathrm{eV}$. The charge transition level is hence $2.2 \mathrm{eV}$ above the pristine VBM. For the $\varepsilon^{+1 / 0}$, we find $\mathrm{E}_{\mathrm{QP}}=0.1 \mathrm{eV}$ and $\mathrm{E}_{\text {relax }}=-0.01 \mathrm{eV}$, giving the charge transition level $\sim 0.1 \mathrm{eV}$ above the VBM. Fig. 5 (c) and (d) show the plot of formation energy with respect to the Fermi level computed using the DFT $+\mathrm{GW}$ formalism. Note that we do not add any electrostatic correction terms here since the quasiparticle excitation energies are taken from the neutral system. The $\varepsilon^{0 /-1}$ computed using hybrid functionals in the literature are $1.9 \mathrm{eV}$ [30] and $1.6 \mathrm{eV}$ [38] above the VBM. The $\varepsilon^{+1 / 0}$ computed using hybrid functionals is found to be below the VBM.

\section{SUBSTRATE SCREENING EFFECTS ON THE CTLS}

The presence of substrates leads to a renormalization of the pristine quasiparticle band gap in $\mathrm{MoS}_{2}$ (Fig. 2 (a)), as well as the term $\mathrm{E}_{\mathrm{QP}}$ in Eqn 3 for the CTL. We compute the renormalization to $\mathrm{E}_{\mathrm{QP}}$ from the super cell calculation. The renormalization to the pristine band gap, on the other hand, is taken from the unit cell calculations (Fig. 2 (a)). We also assume that the $\mathrm{E}_{\text {relax }}$ term is the same in the presence and absence of substrates. Fig. 6 shows the CTLs in the quasiparticle band gap of pristine $\mathrm{MoS}_{2}$ for the various substrates. The $\varepsilon^{+1 / 0}$ is pinned close to the VBM, within $100 \mathrm{meV}$. The defect level involved in this 


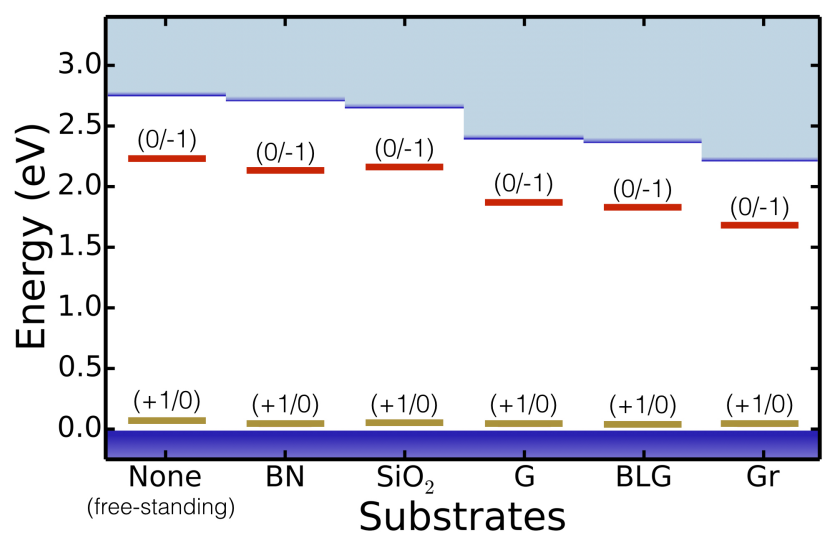

FIG. 6. The $\varepsilon^{+1 / 0}$ and $\varepsilon^{0 /-1}$ charge transition levels of the sulfur vacancy defect computed using the $\mathrm{DFT}+\mathrm{GW}$ formalism. The levels are shown with respect to the valence band maximum of pristine $\mathrm{MoS}_{2}$ in the presence of $\mathrm{BN}$, silica, graphene $(\mathrm{G})$, bilayer graphene (BLG) and graphite (Gr) substrates.

transition is a relatively shallow level with a larger band width (Fig. 3 (a)). The larger band width indicates slight hybridization with the valence band edge. Hence the effect of substrate screening on this level is similar to that of the VBM. This leads to the pinning of $\varepsilon^{+1 / 0}$. The $\varepsilon^{0 /-1}$, with respect to the VBM, is renormalized by about the same amount as the band gap. The anti-bonding character of the empty defect states is similar to that of the CBM of the pristine $\mathrm{MoS}_{2}$ system. [39] The $\varepsilon^{0 /-1}$ is thus pinned about $500 \mathrm{meV}$ below the CBM in the presence of substrates as well as in the free-standing configuration (Fig. 6).

\section{CONCLUSION}

We have studied the effect of substrate screening on the electronic structure of monolayer $\mathrm{MoS}_{2}$. The substrates included in our calculations are: $\mathrm{BN}, \mathrm{SiO}_{2}$, graphene, bilayer graphene and graphite. These substrates lead to a significant renormalization of the quasiparticle band gap of $\mathrm{MoS}_{2}$. In the presence of graphene and graphite substrates, in particular, we find a large reduction of $350 \mathrm{meV}$ and $530 \mathrm{meV}$, respectively. These results are in good agreement with recent experimental measurements on these systems. [32] We have also studied the charge transition levels of sulfur vacancy defects in $\mathrm{MoS}_{2}$ using the DFT+GW formalism. We find two CTLs lying in the pristine quasiparticle band gap of $\mathrm{MoS}_{2}$, the $(+1 / 0)$ and the 
$(0 /-1)$ level. The $(+1 / 0)$ level and $(0 /-1)$ level are found $0.07 \mathrm{eV}$ and $2.14 \mathrm{eV}$ above the pristine VBM, respectively. The stability of the -1 charge state is in good agreement with recent experimental findings. We also compute the CTLs in the presence of substrates. The CTLs show a renormalization similar to that of pristine $\mathrm{MoS}_{2}$ and remain in the pristine band gap of $\mathrm{MoS}_{2}$. With respect to the VBM, the (0/-1) level is renormalized by the same amount as the gap. The (0/-1) level is thus pinned about $500 \mathrm{meV}$ below the CBM for free-standing $\mathrm{MoS}_{2}$ case as well as in the presence of substrates. The $(+1 / 0)$ level, on the other hand, lies less than $100 \mathrm{meV}$ above the VBM in all the cases. The tuning of the defect levels with choice of substrate would aid in tuning the binding of hydrogen at sulfur vacancy sites, which is important to optimize the hydrogen evolution reaction. Chargeddefect scattering from the -1 charged sulfur vacancy can be avoided if the Fermi level of the system is below the computed CTL. This could improve the mobility of carriers in $\mathrm{MoS}_{2}$. The possibility of tuning the CTLs with choice of substrate need not be restricted to $\mathrm{MoS}_{2}$. Other transition metal dichalcogenides and two-dimensional materials could also be expected to show a similar tuning of the defect CTLs.

We thank the Supercomputer Education and Research Centre (SERC) at IISc for providing the computational facilities. Computational facilities from C-DAC's PARAM Yuva II were also used in this work. We thank Felipe H. da Jornada and Diana Y. Qiu for discussions.

[1] K. F. Mak, C. Lee, J. Hone, J. Shan, and T. F. Heinz, Phys. Rev. Lett. 105, 136805 (2010).

[2] A. Splendiani, L. Sun, Y. Zhang, T. Li, J. Kim, C.-Y. Chim, G. Galli, and F. Wang, Nano Letters 10, 1271 (2010).

[3] Q. H. Wang, K. Kalantar-Zadeh, A. Kis, J. N. Coleman, and M. S. Strano, Nat Nano 7, 699 (2012).

[4] B. Radisavljevic, A. Radenovic, J. Brivio, V. Giacometti, and A. Kis, Nat Nano 6, 147 (2011).

[5] K. F. Mak, K. He, J. Shan, and T. F. Heinz, Nat Nano 7, 494 (2012).

[6] K. Roy, M. Padmanabhan, S. Goswami, T. P. Sai, G. Ramalingam, S. Raghavan, and A. Ghosh, Nat Nano 8, 826 (2013).

[7] O. Lopez-Sanchez, D. Lembke, M. Kayci, A. Radenovic, and A. Kis, Nat Nano 8, 497 (2013). 
[8] L. Ye, H. Li, Z. Chen, and J. Xu, ACS Photonics 3, 692 (2016).

[9] H. Li, C. Tsai, A. L. Koh, L. Cai, A. W. Contryman, A. H. Fragapane, J. Zhao, H. S. Han, H. C. Manoharan, F. Abild-Pedersen, J. K. Norskov, and X. Zheng, Nat Mater 15, 48 (2016).

[10] D. Voiry, M. Salehi, R. Silva, T. Fujita, M. Chen, T. Asefa, V. B. Shenoy, G. Eda, and M. Chhowalla, Nano Letters 13, 6222 (2013).

[11] K. Chang, Z. Mei, T. Wang, Q. Kang, S. Ouyang, and J. Ye, ACS Nano 8, 7078 (2014).

[12] J. Hong, Z. Hu, M. Probert, K. Li, D. Lv, X. Yang, L. Gu, N. Mao, Q. Feng, L. Xie, J. Zhang, D. Wu, Z. Zhang, C. Jin, W. Ji, X. Zhang, J. Yuan, and Z. Zhang, Nature Communications 6, $6293(2015)$.

[13] X. Liu, I. Balla, H. Bergeron, G. P. Campbell, M. J. Bedzyk, and M. C. Hersam, ACS Nano 10, 1067 (2016).

[14] R. Addou, L. Colombo, and R. M. Wallace, ACS Applied Materials \& Interfaces 7, 11921 (2015).

[15] Y. Lin, X. Ling, L. Yu, S. Huang, A. L. Hsu, Y.-H. Lee, J. Kong, M. S. Dresselhaus, and T. Palacios, Nano Letters 14, 5569 (2014).

[16] C. Zhang, A. Johnson, C.-L. Hsu, L.-J. Li, and C.-K. Shih, Nano Letters 14, 2443 (2014).

[17] M. K. L. Man, S. Deckoff-Jones, A. Winchester, G. Shi, G. Gupta, A. D. Mohite, S. Kar, E. Kioupakis, S. Talapatra, and K. M. Dani, Scientific Reports 6, 20890 (2016).

[18] K. Hsieh, V. Kochat, X. Zhang, Y. Gong, C. S. Tiwary, P. M. Ajayan, and A. Ghosh, Nano Letters 17, 5452 (2017), pMID: 28786685.

[19] A. Bruix, J. A. Miwa, N. Hauptmann, D. Wegner, S. Ulstrup, S. S. Grønborg, C. E. Sanders, M. Dendzik, A. Grubišić Čabo, M. Bianchi, J. V. Lauritsen, A. A. Khajetoorians, B. Hammer, and P. Hofmann, Phys. Rev. B 93, 165422 (2016).

[20] S. Ghatak, S. Mukherjee, M. Jain, D. D. Sarma, and A. Ghosh, APL Materials 2, 092515 (2014).

[21] Y. L. Huang, Y. Chen, W. Zhang, S. Y. Quek, C.-H. Chen, L.-J. Li, W.-T. Hsu, W.-H. Chang, Y. J. Zheng, W. Chen, and A. T. S. Wee, Nature Communications 6, 6298 (2015).

[22] Z. Zhang, X. Ji, J. Shi, X. Zhou, S. Zhang, Y. Hou, Y. Qi, Q. Fang, Q. Ji, Y. Zhang, M. Hong, P. Yang, X. Liu, Q. Zhang, L. Liao, C. Jin, Z. Liu, and Y. Zhang, ACS Nano 11, 4328 (2017).

[23] S. Ulstrup, A. G. Cabo, J. A. Miwa, J. M. Riley, S. S. Gronborg, J. C. Johannsen, C. Cacho, O. Alexander, R. T. Chapman, E. Springate, M. Bianchi, M. Dendzik, J. V. Lauritsen, P. D. C. 
King, and P. Hofmann, ACS Nano 10, 6315 (2016).

[24] C. Jin, F. A. Rasmussen, and K. S. Thygesen, The Journal of Physical Chemistry C 119, 19928 (2015).

[25] K. T. Winther and K. S. Thygesen, 2D Materials 4, 025059 (2017).

[26] K. Andersen, S. Latini, and K. S. Thygesen, Nano Letters 15, 4616 (2015).

[27] J. Ryou, Y.-S. Kim, S. KC, and K. Cho, Scientific Reports 6, 29184 (2016).

[28] S. Borghardt, J.-S. Tu, F. Winkler, J. Schubert, W. Zander, K. Leosson, and B. E. Kardynał, Phys. Rev. Materials 1, 054001 (2017).

[29] M. Drüppel, T. Deilmann, P. Krüger, and M. Rohlfing, Nature Communications 8, 2117 (2017).

[30] J.-Y. Noh, H. Kim, and Y.-S. Kim, Phys. Rev. B 89, 205417 (2014).

[31] W. Zhou, X. Zou, S. Najmaei, Z. Liu, Y. Shi, J. Kong, J. Lou, P. M. Ajayan, B. I. Yakobson, and J.-C. Idrobo, Nano Letters 13, 2615 (2013).

[32] S. H. Song, M.-K. Joo, M. Neumann, H. Kim, and Y. H. Lee, Nature Communications 8, $2121(2017)$.

[33] Y. Ouyang, C. Ling, Q. Chen, Z. Wang, L. Shi, and J. Wang, Chemistry of Materials 28, 4390 (2016), https://doi.org/10.1021/acs.chemmater.6b01395.

[34] C. Tsai, F. Abild-Pedersen, and J. K. Nrskov, Nano Letters 14, 1381 (2014), pMID: 24499163.

[35] G. Ye, Y. Gong, J. Lin, B. Li, Y. He, S. T. Pantelides, W. Zhou, R. Vajtai, and P. M. Ajayan, Nano Letters 16, 1097 (2016), pMID: 26761422, https://doi.org/10.1021/acs.nanolett.5b04331.

[36] W. Kohn and L. J. Sham, Phys. Rev. 140, A1133 (1965).

[37] P. Hohenberg and W. Kohn, Phys. Rev. 136, B864 (1964).

[38] H.-P. Komsa and A. V. Krasheninnikov, Phys. Rev. B 91, 125304 (2015).

[39] M. G. Sensoy, D. Vinichenko, W. Chen, C. M. Friend, and E. Kaxiras, Phys. Rev. B 95, 014106 (2017).

[40] D. Le, T. B. Rawal, and T. S. Rahman, The Journal of Physical Chemistry C 118, 5346 (2014).

[41] S. KC, R. C. Longo, R. Addou, R. M. Wallace, and K. Cho, Nanotechnology 25, 375703 (2014). 
[42] S. Haldar, H. Vovusha, M. K. Yadav, O. Eriksson, and B. Sanyal, Phys. Rev. B 92, 235408 (2015).

[43] J. P. Perdew and M. Levy, Phys. Rev. Lett. 51, 1884 (1983).

[44] J. Heyd, G. E. Scuseria, and M. Ernzerhof, The Journal of Chemical Physics 118, 8207 (2003).

[45] J. K. Ellis, M. J. Lucero, and G. E. Scuseria, Applied Physics Letters 99, 261908 (2011).

[46] S. Bhattacharyya and A. K. Singh, Phys. Rev. B 86, 075454 (2012).

[47] N. Krane, C. Lotze, J. M. Lger, G. Reecht, and K. J. Franke, Nano Letters 16, 5163 (2016).

[48] M. Jain, J. R. Chelikowsky, and S. G. Louie, Phys. Rev. Lett. 107, 216806 (2011).

[49] P. Rinke, A. Janotti, M. Scheffler, and C. G. Van de Walle, Phys. Rev. Lett. 102, 026402 (2009).

[50] C. Freysoldt, B. Grabowski, T. Hickel, J. Neugebauer, G. Kresse, A. Janotti, and C. G. Van de Walle, Rev. Mod. Phys. 86, 253 (2014).

[51] A. Malashevich, M. Jain, and S. G. Louie, Phys. Rev. B 89, 075205 (2014).

[52] M. Jain, J. R. Chelikowsky, and S. G. Louie, Phys. Rev. Lett. 107, 216803 (2011).

[53] W. Chen and A. Pasquarello, Journal of Physics: Condensed Matter 27, 133202 (2015).

[54] D. Y. Qiu, F. H. da Jornada, and S. G. Louie, Phys. Rev. Lett. 111, 216805 (2013).

[55] A. J. Bradley, M. M. Ugeda, F. H. da Jornada, D. Y. Qiu, W. Ruan, Y. Zhang, S. Wickenburg, A. Riss, J. Lu, S.-K. Mo, Z. Hussain, Z.-X. Shen, S. G. Louie, and M. F. Crommie, Nano Letters 15, 2594 (2015).

[56] D. Y. Qiu, F. H. da Jornada, and S. G. Louie, Phys. Rev. B 93, 235435 (2016).

[57] M. M. Ugeda, A. J. Bradley, S.-F. Shi, F. H. da Jornada, Y. Zhang, D. Y. Qiu, W. Ruan, S.-K. Mo, Z. Hussain, Z.-X. Shen, F. Wang, S. G. Louie, and M. F. Crommie, Nat Mater 13, 1091 (2014).

[58] D. Y. Qiu, F. H. da Jornada, and S. G. Louie, Nano Letters 17, 4706 (2017), pMID: 28677398.

[59] J. Shi, M. Liu, J. Wen, X. Ren, X. Zhou, Q. Ji, D. Ma, Y. Zhang, C. Jin, H. Chen, S. Deng, N. Xu, Z. Liu, and Y. Zhang, Advanced Materials 27, 7086 (2015).

[60] C.-I. Lu, C. J. Butler, J.-K. Huang, C.-R. Hsing, H.-H. Yang, Y.-H. Chu, C.-H. Luo, Y.-C. Sun, S.-H. Hsu, K.-H. O. Yang, C.-M. Wei, L.-J. Li, and M.-T. Lin, Applied Physics Letters 106, $181904(2015)$. 
[61] K. Yao, A. Yan, S. Kahn, A. Suslu, Y. Liang, E. S. Barnard, S. Tongay, A. Zettl, N. J. Borys, and P. J. Schuck, Phys. Rev. Lett. 119, 087401 (2017).

[62] M.-H. Chiu, C. Zhang, H.-W. Shiu, C.-P. Chuu, C.-H. Chen, C.-Y. S. Chang, C.-H. Chen, M.-Y. Chou, C.-K. Shih, and L.-J. Li, Nature Communications 6, 7666 (2015).

[63] P. Giannozzi, S. Baroni, N. Bonini, M. Calandra, R. Car, C. Cavazzoni, D. Ceresoli, G. L. Chiarotti, M. Cococcioni, I. Dabo, A. D. Corso, S. de Gironcoli, S. Fabris, G. Fratesi, R. Gebauer, U. Gerstmann, C. Gougoussis, A. Kokalj, M. Lazzeri, L. Martin-Samos, N. Marzari, F. Mauri, R. Mazzarello, S. Paolini, A. Pasquarello, L. Paulatto, C. Sbraccia, S. Scandolo, G. Sclauzero, A. P. Seitsonen, A. Smogunov, P. Umari, and R. M. Wentzcovitch, Journal of Physics: Condensed Matter 21, 395502 (2009).

[64] M. H. Naik and M. Jain, Computer Physics Communications 226, 114 (2018).

[65] J. Deslippe, G. Samsonidze, D. A. Strubbe, M. Jain, M. L. Cohen, and S. G. Louie, Computer Physics Communications 183, 1269 (2012).

[66] M. Rohlfing and S. G. Louie, Phys. Rev. B 62, 4927 (2000).

[67] M. S. Hybertsen and S. G. Louie, Phys. Rev. B 34, 5390 (1986).

[68] D. Y. Qiu, F. H. da Jornada, and S. G. Louie, Phys. Rev. Lett. 115, 119901 (2015).

[69] G. Samsonidze, M. Jain, J. Deslippe, M. L. Cohen, and S. G. Louie, Phys. Rev. Lett. 107, 186404 (2011).

[70] A. Stathopoulos and J. R. McCombs, ACM Transactions on Mathematical Software 37, 21:1 (2010).

[71] L. Wu, E. Romero, and A. Stathopoulos, SIAM J. Sci. Comput., to appear abs/1607.01404 (2016).

[72] L. S. Blackford, J. Choi, A. Cleary, E. D’Azevedo, J. Demmel, I. Dhillon, J. Dongarra, S. Hammarling, G. Henry, A. Petitet, K. Stanley, D. Walker, and R. C. Whaley, ScaLAPACK Users' Guide (Society for Industrial and Applied Mathematics, Philadelphia, PA, 1997).

[73] J. Deslippe, G. Samsonidze, M. Jain, M. L. Cohen, and S. G. Louie, Phys. Rev. B 87, 165124 (2013).

[74] S. Ismail-Beigi, Phys. Rev. B 73, 233103 (2006).

[75] S. Grimme, Journal of Computational Chemistry 27, 1787 (2006).

[76] M. H. Naik and M. Jain, Phys. Rev. B 95, 165125 (2017). 
[77] H. Coy Diaz, J. Avila, C. Chen, R. Addou, M. C. Asensio, and M. Batzill, Nano Letters 15, $1135(2015)$.

[78] D. Pierucci, H. Henck, J. Avila, A. Balan, C. H. Naylor, G. Patriarche, Y. J. Dappe, M. G. Silly, F. Sirotti, A. T. C. Johnson, M. C. Asensio, and A. Ouerghi, Nano Letters 16, 4054 (2016).

[79] M. Rohlfing, Phys. Rev. Lett. 108, 087402 (2012).

[80] J. B. Neaton, M. S. Hybertsen, and S. G. Louie, Phys. Rev. Lett. 97, 216405 (2006).

[81] K. S. Thygesen and A. Rubio, Phys. Rev. Lett. 102, 046802 (2009).

[82] Y. Liang and L. Yang, Phys. Rev. Lett. 114, 063001 (2015).

[83] G. Samsonidze, M. L. Cohen, and S. G. Louie, Computational Materials Science 91, 187 (2014).

[84] H. Schweiger, P. Raybaud, G. Kresse, and H. Toulhoat, Journal of Catalysis 207, 76 (2002).

[85] M. V. Bollinger, K. W. Jacobsen, and J. K. Nørskov, Phys. Rev. B 67, 085410 (2003). 